\title{
ESTUDIO DE FLUIDOS NO NEWTONIANOS CON LOS MÉTODOS DE VOLÚMENES Y ELEMENTOS FINITOS
}

\author{
Guillermo Sánchez M. ${ }^{1} \quad$ César Vial R. $^{2} \quad$ Nelson Moraga B. ${ }^{2}$ \\ Recibido el 16 de enero de 2002, aceptado el 20 de agosto de 2002
}

\begin{abstract}
RESUMEN
En el presente trabajo, se realiza un estudio numérico de mecánica de fluidos 2D en una contracción brusca para fluidos no newtonianos tipo ley de potencia de Ostwald-de-Waele. Se emplean dos métodos de resolución, el método de los volúmenes finitos y el método de los elementos finitos. En el primer método se utiliza el algoritmo SIMPLE, para la resolución de las ecuaciones de momentum lineal y de continuidad. Para el método de elementos finitos se utiliza un código propio en lenguaje Fortran 90. En ambos métodos se estudia el comportamiento del fluido para diferentes índices de potencia. Los cálculos se realizan con malla variable rectangular (método de volúmenes finitos) y con malla apegada al contorno (método elementos finitos).
\end{abstract}

Palabras claves: Fluido no-newtoniano, método de elementos finitos, método de volúmenes finitos

\begin{abstract}
In the present work, a numerical study of 2D fluid mechanics in an abrupt contraction is made, for non-newtonian flow with Ostwald-de Waele type law of power. Two solution methods are used: finite volumes and finite elements methods. In the finite volumes method, the SIMPLE algorithm is used to solve the linear momentum and continuity equations. In the finite elements method, Fortran 90 language is used for writing our own program. In both methods, the behavior of the fluid, for different indexes of power, is studied. The calculations are made with rectangular variable mesh (method of finite volumes) and with mesh attached to the contour (method of finite elements).
\end{abstract}

Keywords: Non-Newtonian flow, finite elements method, finite volumes method

\section{INTRODUCCIÓN}

Prácticamente la totalidad de los fluidos utilizados en plantas industriales corresponden a fluidos no newtonianos, los cuales son tratados o calculados según leyes para flujos newtonianos, (velocidad proporcional al esfuerzo de corte). Se ha mostrado en estudios previos, el error que se comete al tratar como newtonianos, fluidos dilatantes o seudo-plásticos, de aquí la importancia del desarrollo de herramientas para el estudio de estos fluidos.

En el presente trabajo se modela el flujo permanente, isotérmico de fluido no newtoniano tipo ley de potencia de Ostwald-de-Waele con el método de los volúmenes finitos y con el método de los elementos finitos.

El modelo de ley de potencia de Ostwald-de-Waele puede modelar fluidos seudoplásticos cuando el exponente, que caracteriza la ley, es mayor que uno. Algunos ejemplos de fluidos seudoplásticos son: pinturas, gomas, sangre, suspensiones, etcétera. Esta ley modela los fluidos newtonianos cuando $n$ es igual a uno, y cuando $n$ es menor que uno, representa a fluidos dilatantes. Como ejemplos de estos fluidos, se pueden citar, entre otros, el azúcar, tierra húmeda, soluciones en bórax.

\section{DESCRIPCIÓN DEL PROBLEMA}

Se estudia el movimiento de un fluido no newtoniano en un ducto circular con un cambio brusco de sección transversal, como se muestra la Fig. 1. La condición de borde a la entrada, en $\mathrm{z}=0$, considera un perfil parabólico de velocidades. El flujo a la salida, $\mathrm{z}=\mathrm{L}$, es desarrollado. En el centro de la tubería, $r=0$, se supone simetría y en las paredes se postula que no hay

\footnotetext{
1 Universidad de Santiago de Chile, Departamento de Matemática y C.C., Casilla 307, Correo 2, Santiago, Chile, gsanchez@ usach.cl

2 Universidad de Santiago de Chile, Departamento de Ingeniería Mecánica, Casilla 307, Correo 2, Santiago, Chile, nmoraga@ lauca.usach.cl
} 
deslizamiento, $\mathrm{u}=\mathrm{v}=0$. La modelación se realizó considerando los índices de potencia, $\mathrm{n}=0.75, \mathrm{n}=1.0 \mathrm{y}$ $\mathrm{n}=1.25$. El número de Raynold considerado en todos los casos fue $\mathrm{Re}=0.4$.

\section{MODELO MATEMÁTICO}

En este trabajo, se considera un flujo permanente, laminar, bidimensional, isotérmico y fluido incompresible no newtoniano tipo ley de potencia.

Con las características expuestas, las ecuaciones del modelo matemático se reducen a:

Ecuación de continuidad:

$$
\frac{\partial \mathrm{u}}{\partial \mathrm{z}}+\frac{\mathrm{v}}{\mathrm{r}}+\frac{\partial \mathrm{v}}{\partial \mathrm{r}}=0
$$

Ecuación de momento lineal en z:

$$
\rho\left(u \frac{\partial u}{\partial z}+v \frac{\partial u}{\partial r}\right)=-\frac{\partial p}{\partial z}+\mu\left(\frac{\partial^{2} u}{\partial z^{2}}+\frac{1}{r} \frac{\partial u}{\partial r}+\frac{\partial^{2} u}{\partial r^{2}}\right)
$$

Ecuación de momento lineal en r:

$$
\rho\left(u \frac{\partial v}{\partial z}+v \frac{\partial v}{\partial r}\right)=-\frac{\partial p}{\partial r}+\mu\left(\frac{\partial^{2} v}{\partial z^{2}}-\frac{v}{r^{2}}+\frac{1}{r} \frac{\partial v}{\partial r}+\frac{\partial^{2} v}{\partial r^{2}}\right)(3)
$$

donde, los esfuerzos de corte normales y tangenciales se han determinado en función de la viscosidad efectiva mediante:

$$
\begin{aligned}
& \sigma_{z z}=2 \eta \frac{\partial u}{\partial z}, \\
& \sigma_{r r}=2 \eta \frac{\partial v}{\partial z}, \\
& \tau_{r r z}=\tau_{z r}=\eta\left(\frac{\partial u}{\partial r}+\frac{\partial v}{\partial z}\right) .
\end{aligned}
$$

El modelo de ley de potencia define la viscosidad efectiva como:

$$
\eta=\mu\left(\left(\frac{\partial u}{\partial r}\right)^{2}+\left(\frac{\partial v}{\partial z}\right)^{2} \cdot\right)^{\frac{n-1}{2}}
$$

donde $n$ es el índice de potencia y $\mu$ es el índice de consistencia. Notar que cuando $n=1, \mathrm{v}$ representa la viscosidad dinámica newtoniana $\mathrm{y}$ el fluido es newtoniano.

Las condiciones de borde para el problema son:

- Condición de no-deslizamiento de paredes rígidas del ducto: $\mathrm{R}_{\mathrm{i}}=\mathrm{R}_{\mathrm{i}}(\mathrm{z})$.

$$
\begin{aligned}
& \mathrm{u}\left(\mathrm{R}_{\mathrm{i}}, \mathrm{z}\right)=0 . \\
& \mathrm{v}\left(\mathrm{R}_{\mathrm{i}}, \mathrm{z}\right)=0 .
\end{aligned}
$$

- Condición de simetría en $\mathrm{R}=0$ :

$$
\begin{aligned}
& \left.\frac{\partial \mathrm{u}}{\partial \mathrm{r}}\right|_{\mathrm{r}=0}=0 . \\
& \mathrm{v}(0, \mathrm{z})=0 .
\end{aligned}
$$

- Perfil parabólico de velocidad en la entrada:

$$
\mathrm{v}(\mathrm{r}, 0)=0
$$

$$
\mathrm{u}(\mathrm{r}, 0)=2 \cdot \mathrm{U}_{\text {med }}\left[1-\left(\frac{\mathrm{r}}{\mathrm{R}}\right)^{2}\right]
$$

donde $\mathrm{U}_{\text {med }}$ es la velocidad media en la entrada del ducto. Hay que notar que este perfil corresponde a un fluido newtoniano y que los cálculos se realizan con este perfil en la entrada, de manera de tener una condición de borde similar.

- Condición de flujo hidrodinámicamente desarrollado al final del ducto:

$$
\begin{aligned}
& \left.\frac{\partial \mathrm{u}}{\partial \mathrm{z}}\right|_{\mathrm{z}=\mathrm{L}}=0 \\
& \mathrm{v}(\mathrm{r}, \mathrm{L})=0
\end{aligned}
$$

\section{ESTRATEGIA DE SOLUCIÓN DEL MVF}

La solución del sistema de ecuaciones diferenciales se solucionó mediante el método de los volúmenes finitos empleando el algoritmo SIMPLE, método semiimplícito de ecuaciones ligadas por la presión, Patankar (1980). 


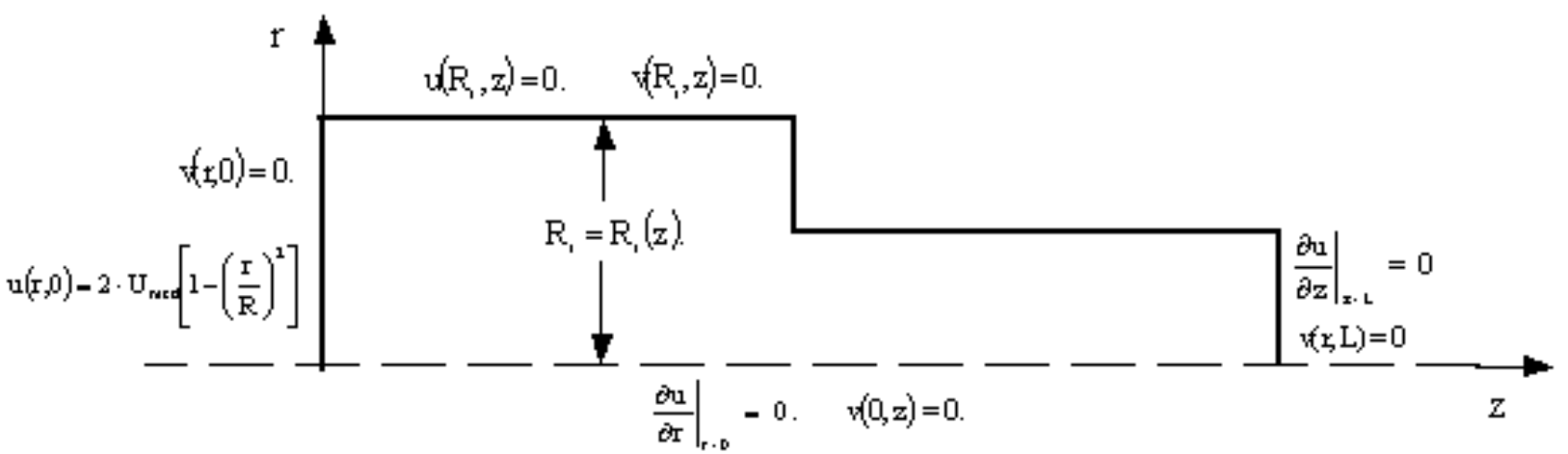

Fig. 1.- Situación física del problema

El sistema de ecuaciones lineales asociado a la discretización del dominio es resuelto con un método híbrido, que utiliza el algoritmo de Thomas TDMA (método directo) y el método iterativo de Gauss-Seidel con relajación sucesiva.

El método para resolver el sistema no lineal formado por el comportamiento no newtoniano del fluido se resuelve empleando la siguiente estrategia:

1. En la primera iteración se resuelve el problema considerando el fluido como newtoniano. Se calculan las velocidades u y v para un campo de presiones dado.

2. Con el campo de velocidades se calcula la viscosidad aparente, en este punto se calcula el campo de velocidades para el fluido no newtoniano.

3. Se repite el paso 2 hasta obtener una convergencia de los valores de la velocidad, el criterio de convergencia utilizado es $S M A X<10^{-6}$, donde $S M A X$ es la mayor creación o destrucción de materia para los volúmenes finitos del dominio.

\section{DISCRETIZACIÓN DEL DOMINIO MVF}

Para simular el manto del cilindro, se utilizan funciones que lo definen y el programa de cálculo asigna una velocidad fija nula fuera de este borde.
Con el fin de realizar cálculos más refinados en el centro y en la salida del ducto, se utiliza una malla variable, la que se muestra en la Fig. 2.

Las dimensiones nominales del ducto son: largo ducto mayor $12[\mathrm{~cm}]$, largo ducto menor $6[\mathrm{~cm}]$, radio mayor $4.0[\mathrm{~cm}]$ y radio menor $1[\mathrm{~cm}]$. Las propiedades del fluido son: viscosidad dinámica $\mu=1[\mathrm{gr} /(\mathrm{cm}(\mathrm{s})]$, densidad $\rho=1\left[\mathrm{gr} / \mathrm{cm}^{3}\right]$, velocidad media $U \_$med $=0.5[\mathrm{~cm} / \mathrm{s}]$. Se realizaron los cálculos para fluido dilatante con índice de potencia $\mathrm{n}=0.75$, para fluido newtoniano, $\mathrm{n}=1$ y para fluido seudo-plástico con índice de potencia $n=1.25$.

\section{ESTRATEGIA DE SOLUCIÓN MEF}

Desde el punto de vista del método de los elementos finitos, las ecuaciones (1) a (7) originan las conocidas formulaciones mixtas en variable primal que, en este caso, son la velocidad $\boldsymbol{u}$ y la presión $P$. En este tipo de formulaciones, la presión juega el rol de los multiplicadores de Lagrange. En forma compacta se obtiene un sistema de la forma:

$$
\left(\begin{array}{cc}
k & C \\
C^{T} & 0
\end{array}\right)\left(\begin{array}{l}
\boldsymbol{u} \\
P
\end{array}\right)=\left(\begin{array}{l}
f \\
0
\end{array}\right)
$$

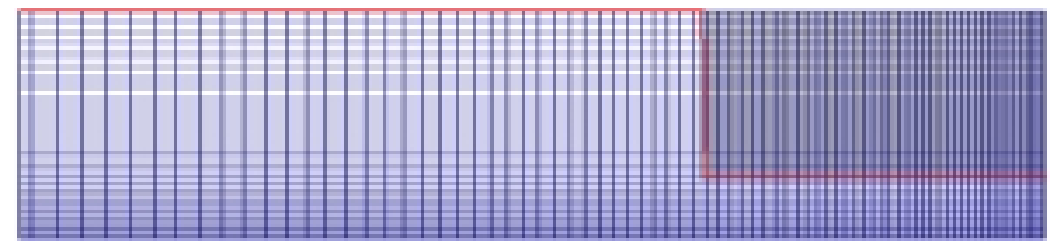

Fig. 2.- Malla variable 80x80 nodos MVF 
Es sabido que al aplicar a nuestro problema, la forma estándar de Galerkin del método de elemento finito, se tienen dificultades al considerar combinaciones arbitrarias para la interpolación de presiones y velocidades [1]. Al tomar un orden menor en la interpolación de la presión que para la velocidad, se obtienen soluciones estables para la formulación de Galerkin del método de elementos finitos para fluidos incompresibles. Con este fin se desarrollaron dos tipos de elementos: a)los elementos con presión continua, elementos de [2], y b) los elementos con presión discontinua, elementos de [3]. Para el caso de las formulaciones del tipo mixto, los elementos que se utilizan, son aquellos que satisfacen la condición de Babuska--Brazzi. Detalles de estos elementos pueden encontrarse en los trabajos de Loula, Franca y Karam. El sistema de ecuaciones resultantes de este tipo de formulaciones no es definida positiva, lo que genera ciertas complicaciones computacionales. Con el objetivo de evitar estas dificultades, se utiliza una formulación basada en un método penalizado, la que puede obtenerse expresando los multiplicadores de Lagrange en términos de las variables primales, esto es:

$$
P=\lambda f(u, v)
$$

donde $\lambda$ es el parámetro de penalización. Cuando se usa la integración gaussiana reducida, puede observarse que en los puntos de integración, los campos de presión concuerdan con el término penalizado obtenido usando la integración reducida. De esta manera, la formulación mixta para nuestro problema adopta la forma:

$$
\left(\begin{array}{ll}
K_{S} & Q \\
Q^{T} & C
\end{array}\right)\left(\begin{array}{l}
\boldsymbol{u} \\
P
\end{array}\right)=\left(\begin{array}{l}
f \\
0
\end{array}\right)
$$

donde:

$$
\begin{aligned}
& K_{S}=\int_{\Omega} B^{T} 2 \mu B d \Omega \\
& Q=\int_{\Omega} B^{T} \Theta^{T} M d \Omega \\
& C=\int_{\Omega} \lambda B^{T} \Theta{ }^{T} B d \Omega
\end{aligned}
$$

La variable $P$ puede eliminarse, a nivel de cada elemento, usando la siguiente formulación de penalización:

$$
\begin{aligned}
& {\left[K_{S}+Q C^{-1} Q^{T}\right]_{e} \bar{v}_{e}=f_{e}} \\
& \bar{P}_{e}=\left(C^{-1} Q^{T}\right)_{e} \bar{v}_{e}
\end{aligned}
$$

Por lo tanto, las ecuaciones globales pueden expresarse como:

$$
K \bar{v}=F
$$

donde $v$ son las velocidades y $F$ son las fuerzas totales incluidas las fuerzas generalizada del cuerpo. Reiteramos que el término $\lambda$ debe ser evaluado usando integración reducida.

\section{DISCRETIZACIÓN DEL DOMINIO}

Para la discretización espacial del dominio se usó el generador de mallas EASYMESH, y un programa desarrollado en FORTRAN 90.

La malla utilizada se muestra en la Fig. 3. Notar que se refina la discretización cerca del manto y en el vértice de la contracción.

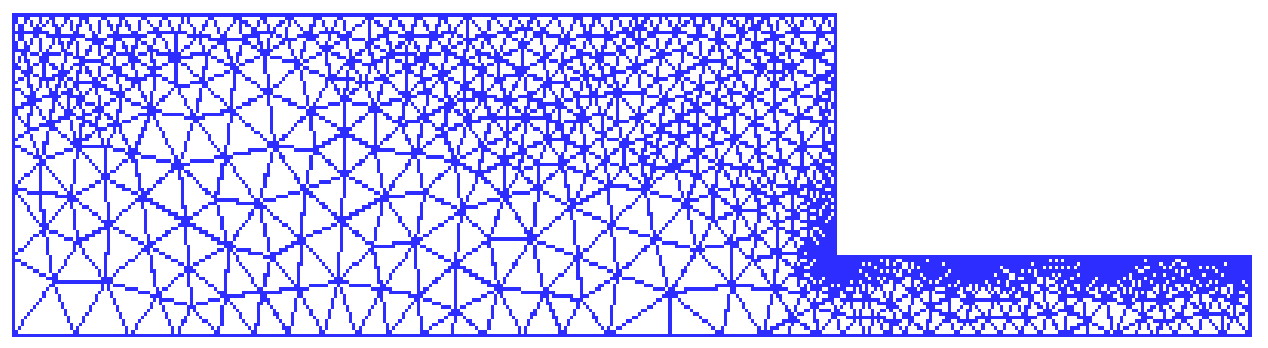

Fig. 3.- Malla de elementos triangulares cuadráticos 



\section{PRESENTACIÓN Y ANÁLISIS DE RESULTADOS}

Como se observa en las Figs. 4 y 5, se obtuvo las líneas de corriente para cada uno de casos estudiados, pudiéndose notar una gran similitud para las situaciones en estudio obtenidas con cada método.

También se obtuvo el desarrollo de los perfiles de velocidad $u$, a lo largo del ducto, como muestran las Figs. 6 a 8, en donde se observa que el comportamiento de los fluidos en este caso, es similar.

El desarrollo de la velocidad $v$ a lo largo del ducto se observa en las Figs. 9 y 10, notándose que éste, permanece para cada método de resolución independiente del índice de potencia y en general la velocidad mínima $\mathrm{V}$ para el método de los volúmenes finitos, es el doble en valor absoluto, que la velocidad mínima para el método de los elementos finitos.

También se realizó la comparación del desarrollo de la velocidad en el centro del ducto para los distintos casos estudiados, como se observa en las Figs. 9, 10 y 11, encontrando un comportamiento similar en ambos métodos.

En el cálculo con elementos finitos, la variación de la velocidad en el centro, se produce antes que en el caso de los volúmenes finitos, pero en general, siguen el mismo comportamiento.

Se puede observar que las velocidades máximas en la salida del ducto, son prácticamente similares para los dos métodos con un índice de potencia dado.

Una de las características que diferencia a los fluidos no-newtonianos de los newtonianos, es el desarrollo del perfil de velocidades, en las Figs. 5 y 6 se observa el desarrollo total del perfil a lo largo del ducto, en la Figs. 12 a 17, se observa el comportamiento del perfil en la salida del ducto, notándose una apreciable similitud.

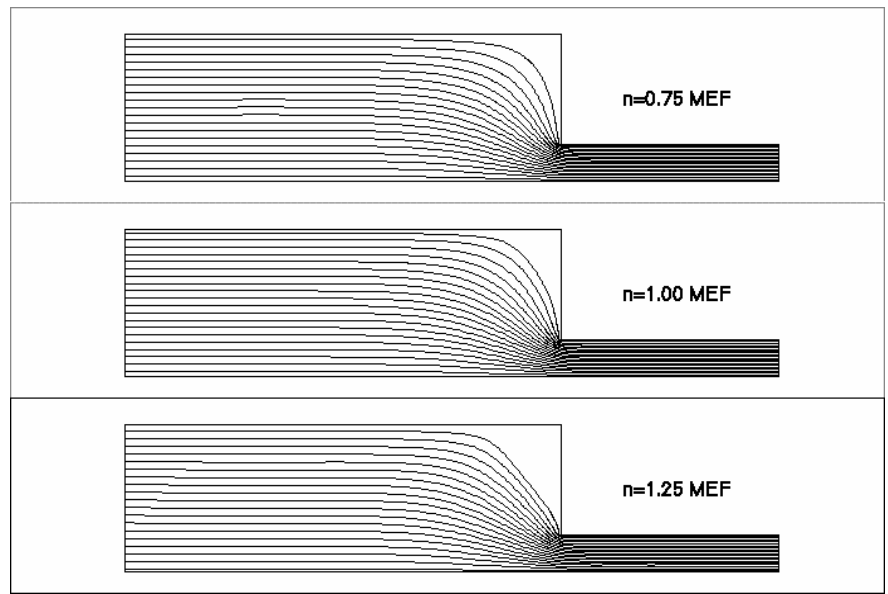

Fig. 4.- Líneas de corriente con MEF

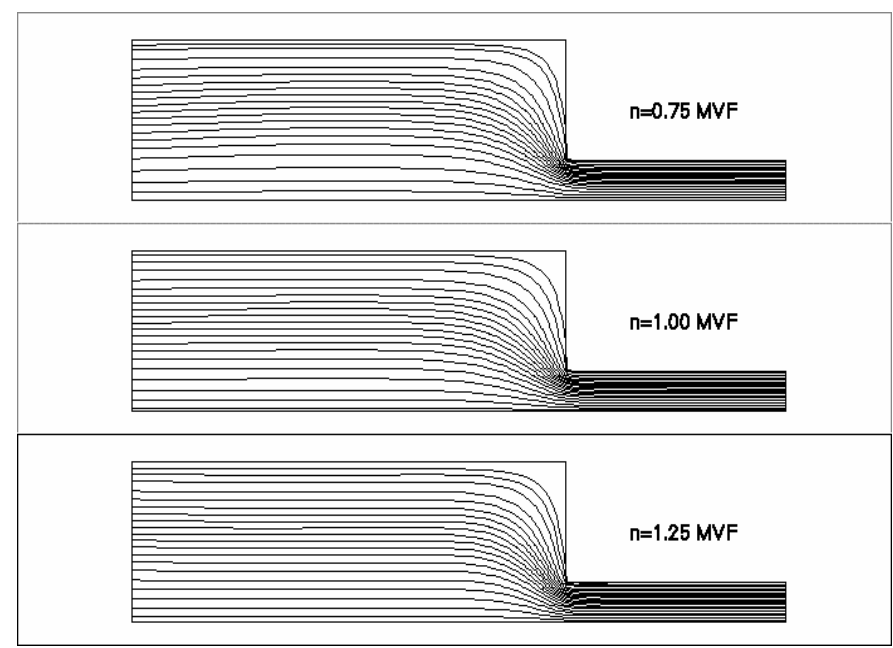

5.- Líneas de corriente con MVF 


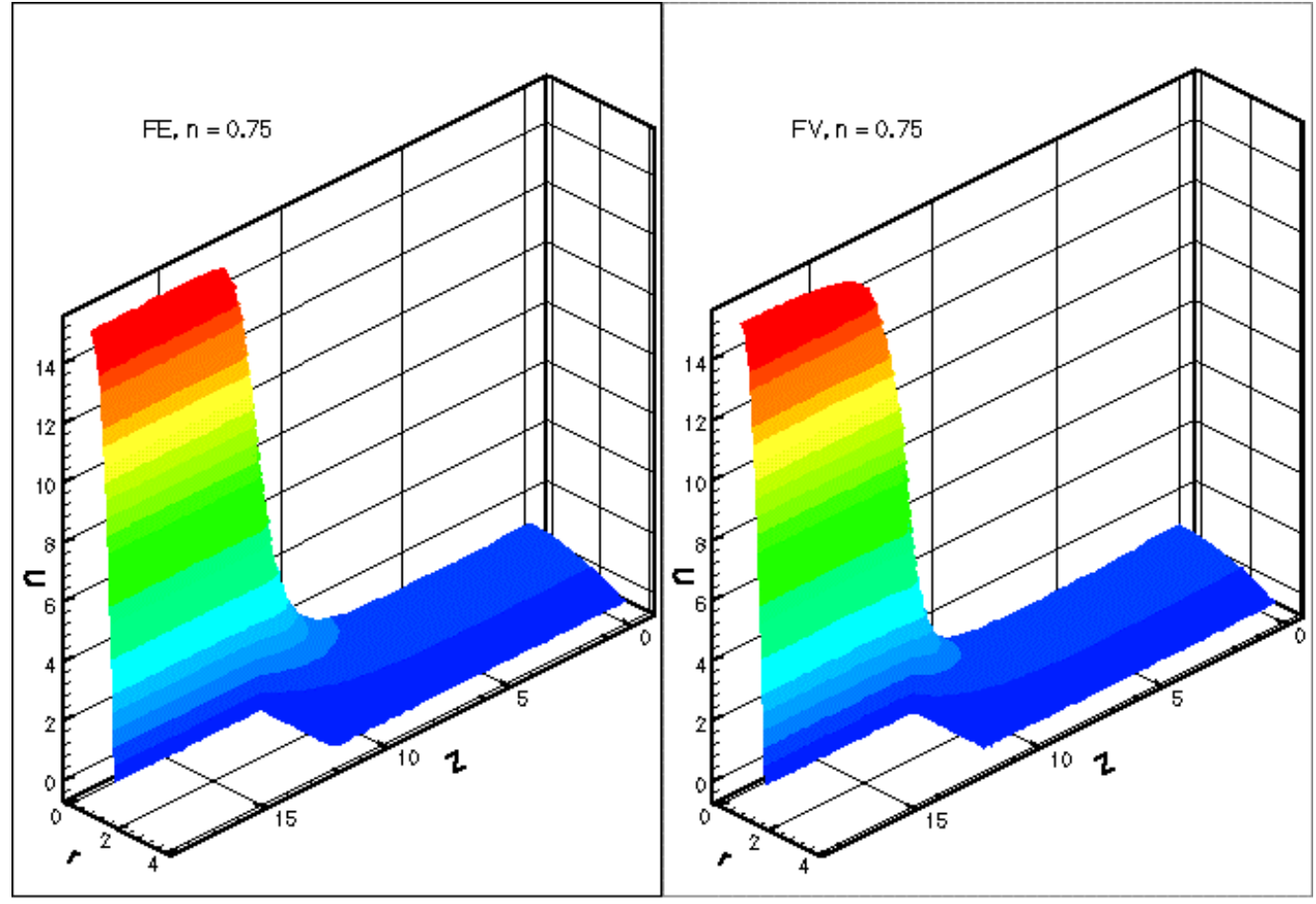

Fig. 6.- Variación en la dirección del flujo

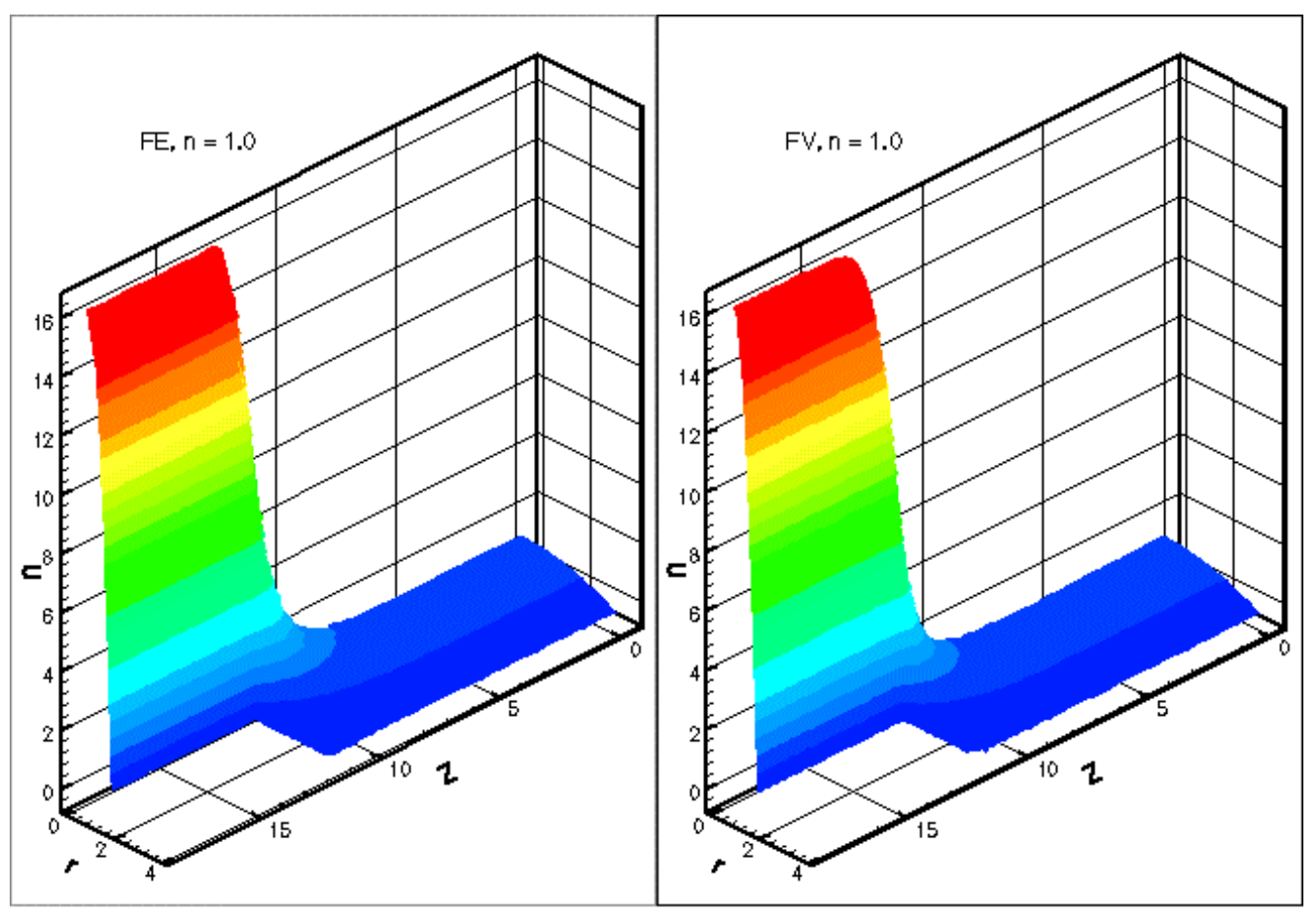

Fig. 7.- Variación en la dirección del flujo 


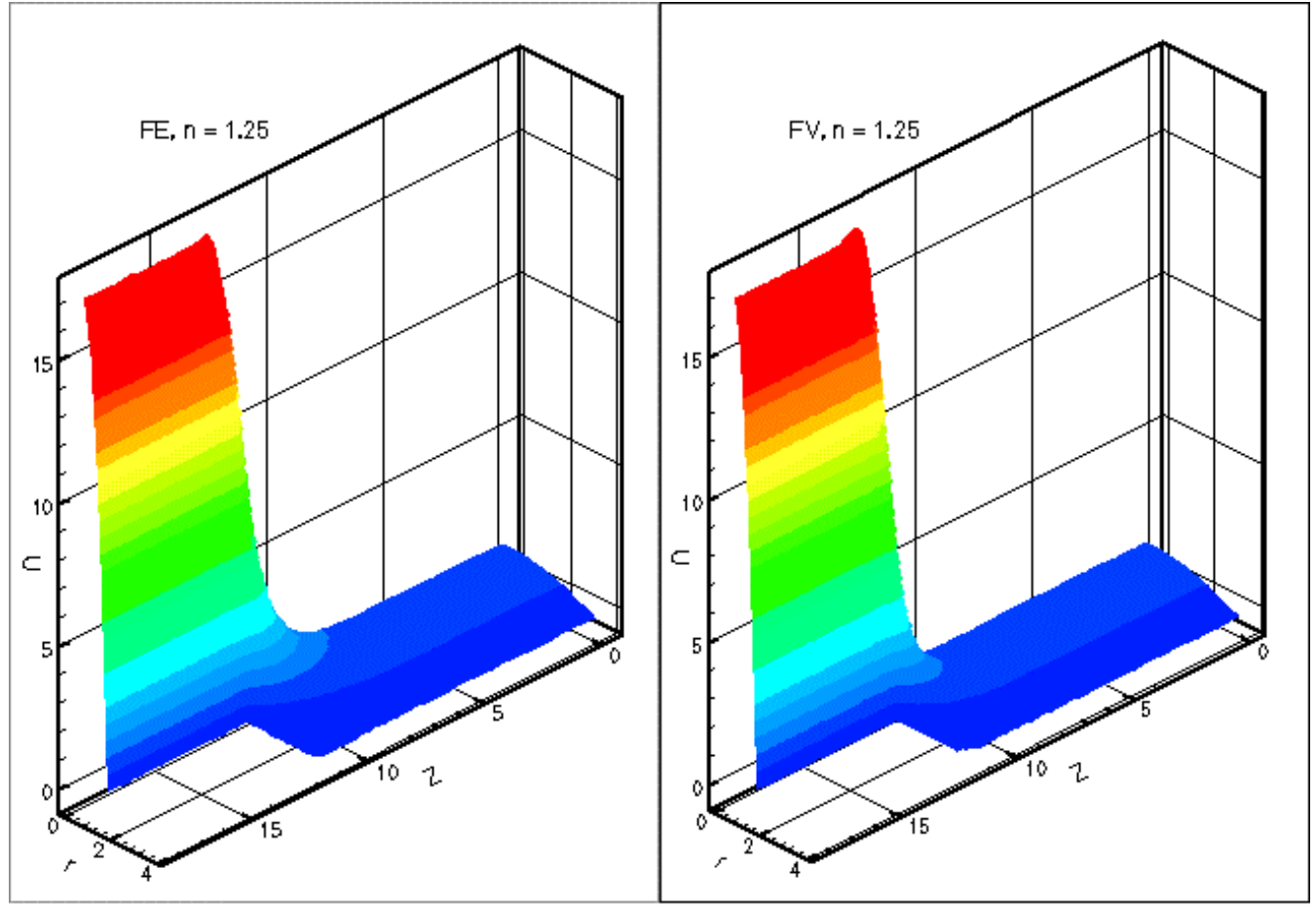

Fig. 8.- Variación en la dirección del flujo

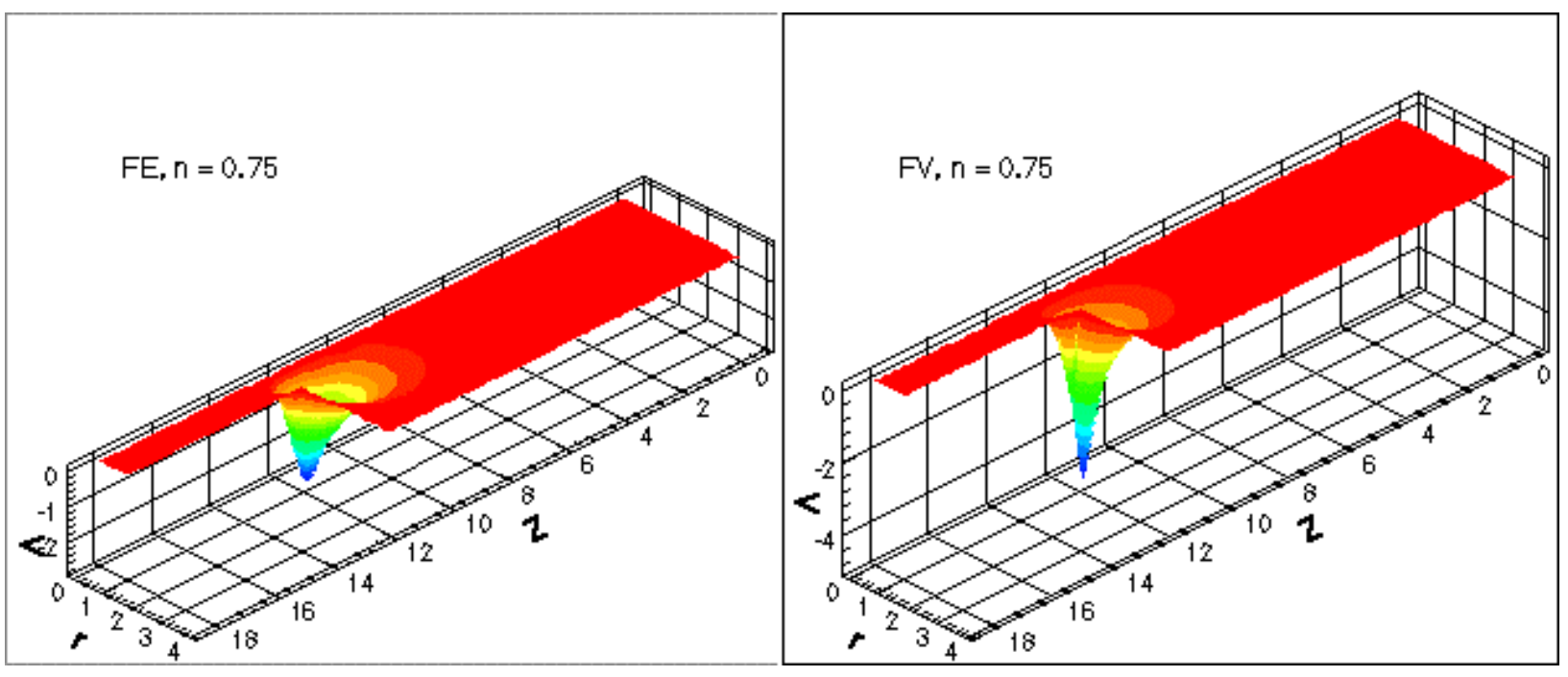

Fig. 9.- Variación en la dirección del flujo 


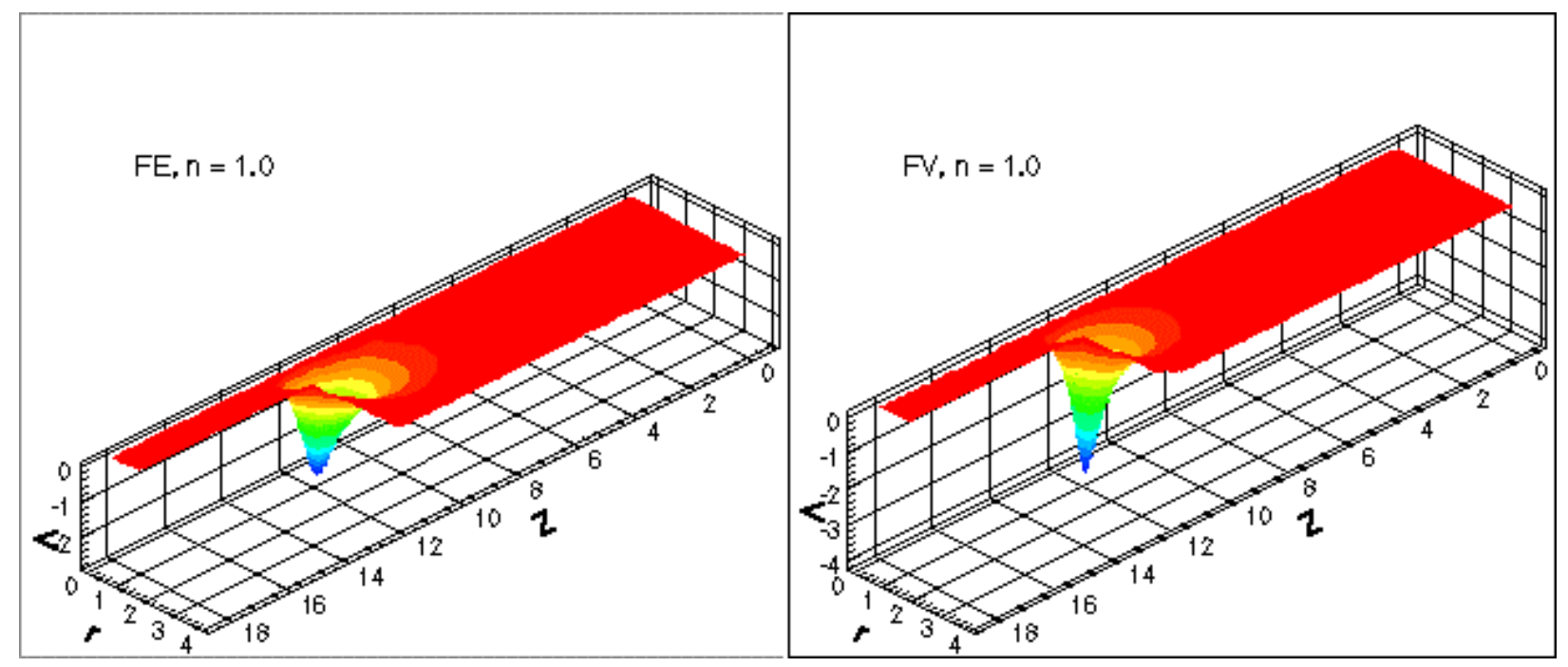

Fig. 10.- Variación en la dirección del flujo

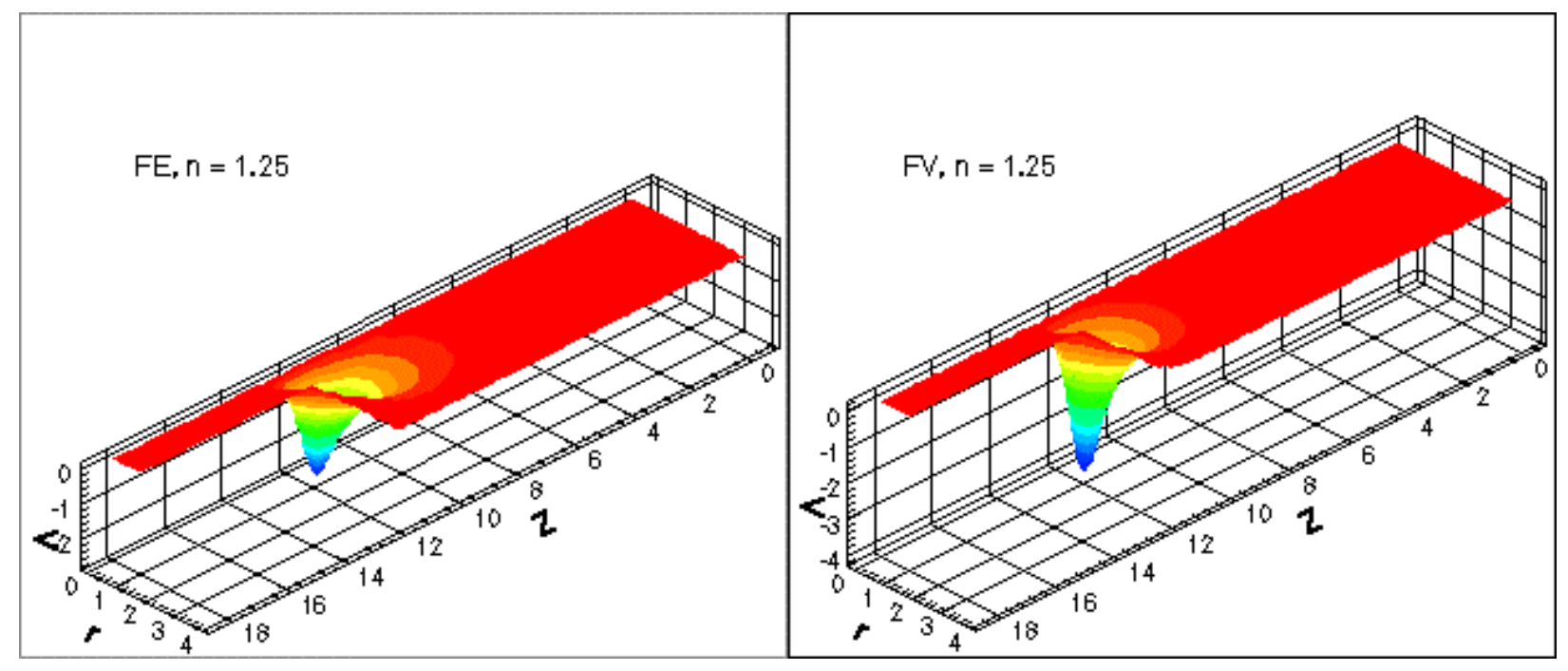

Fig. 11.- Variación en la dirección del flujo 


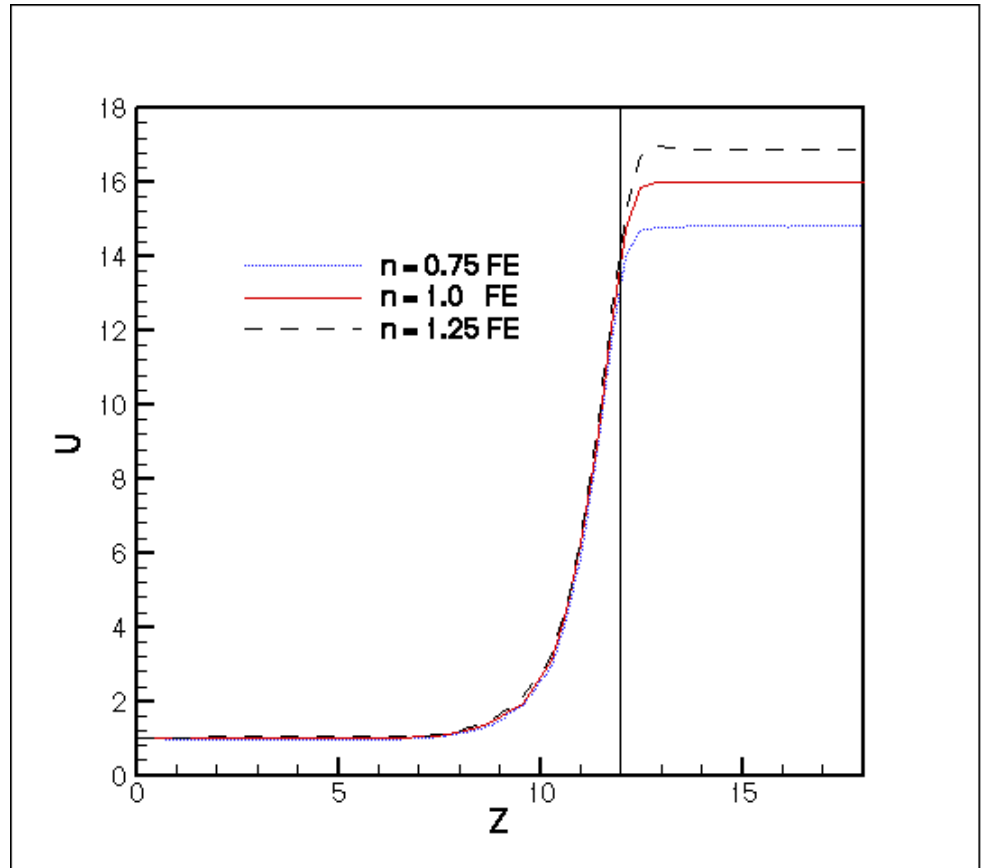

Fig. 12.- Desarrollo de la velocidad en el centro del ducto con MEF

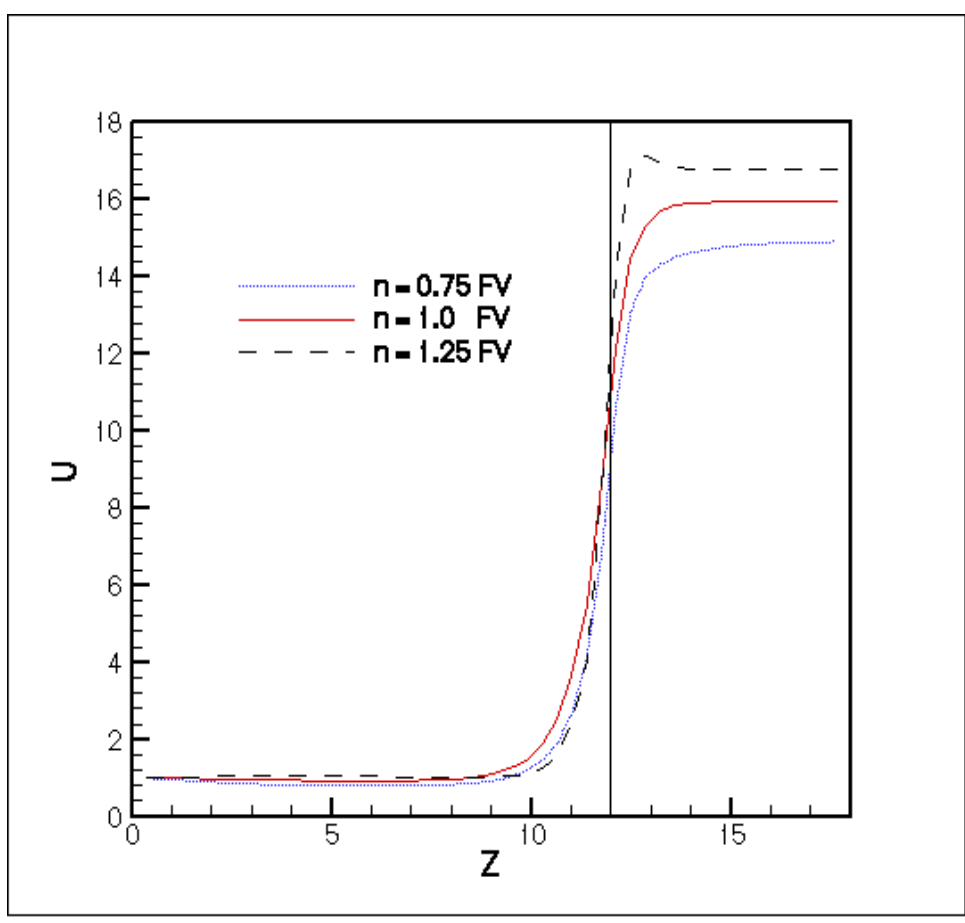

Fig. 13.- Desarrollo de la velocidad en el centro del ducto con MVF 


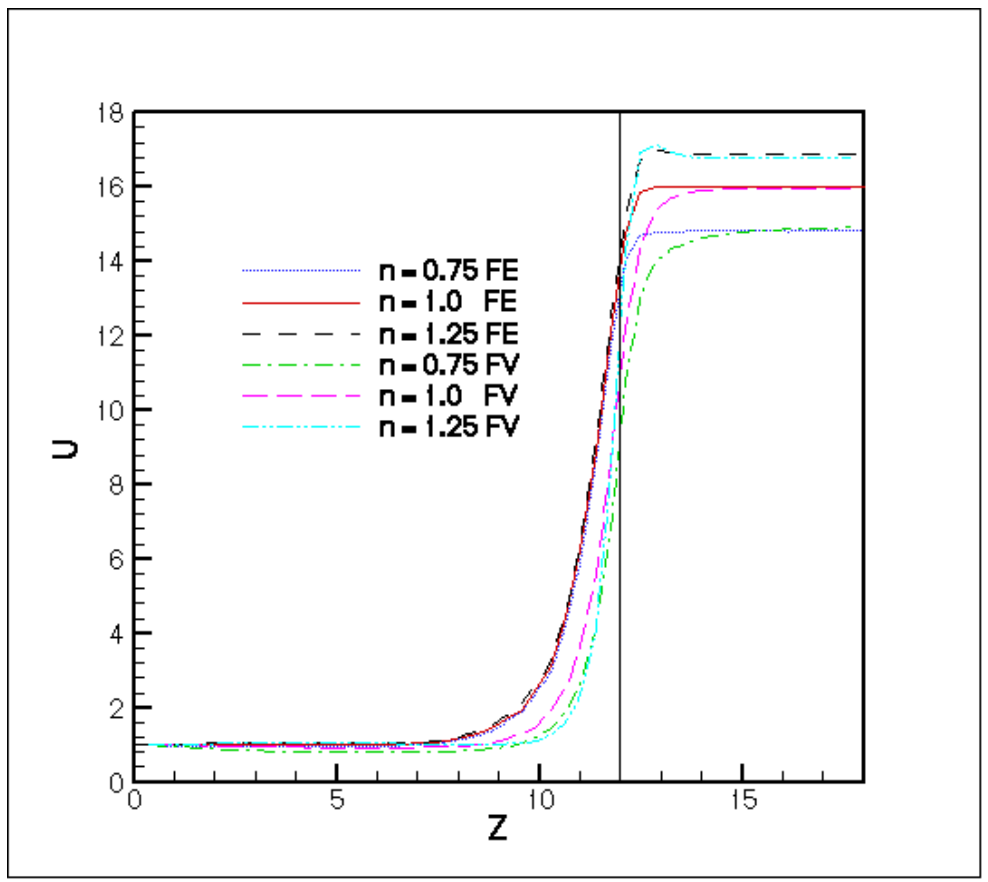

Fig. 14.- Comparación de la velocidad en el centro del ducto

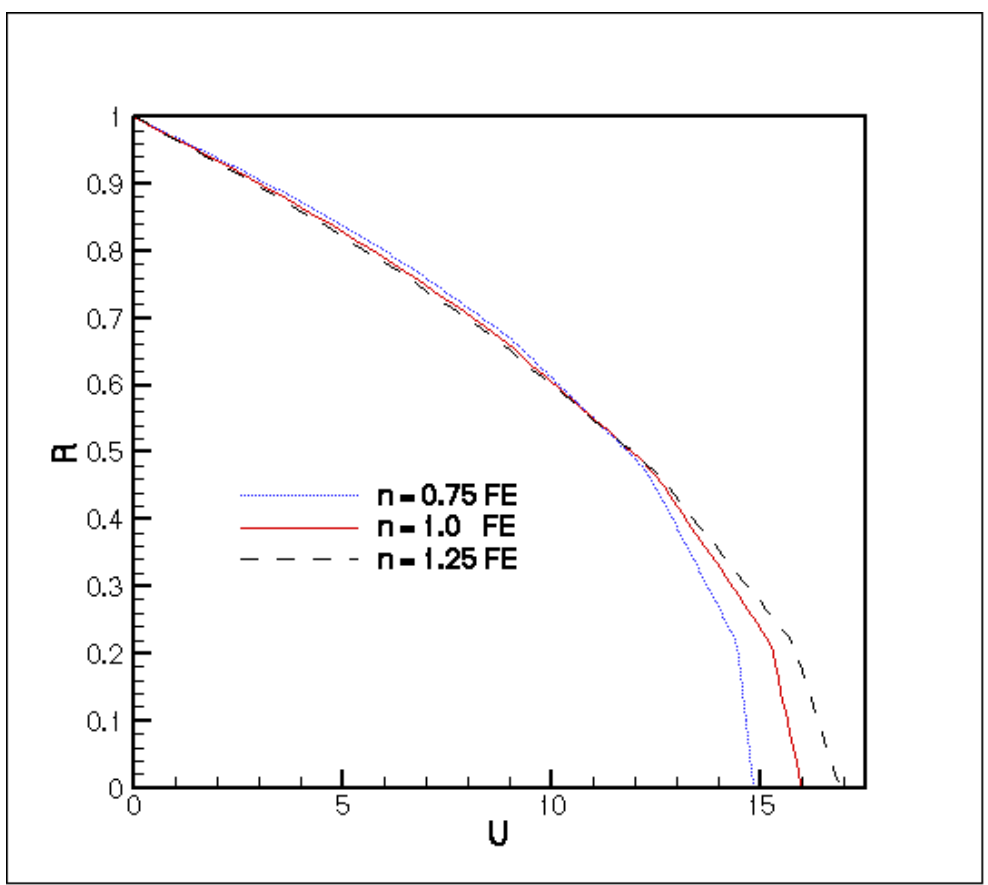

Fig. 15.- Perfil de la velocidad $u$ a la salida con MEF 


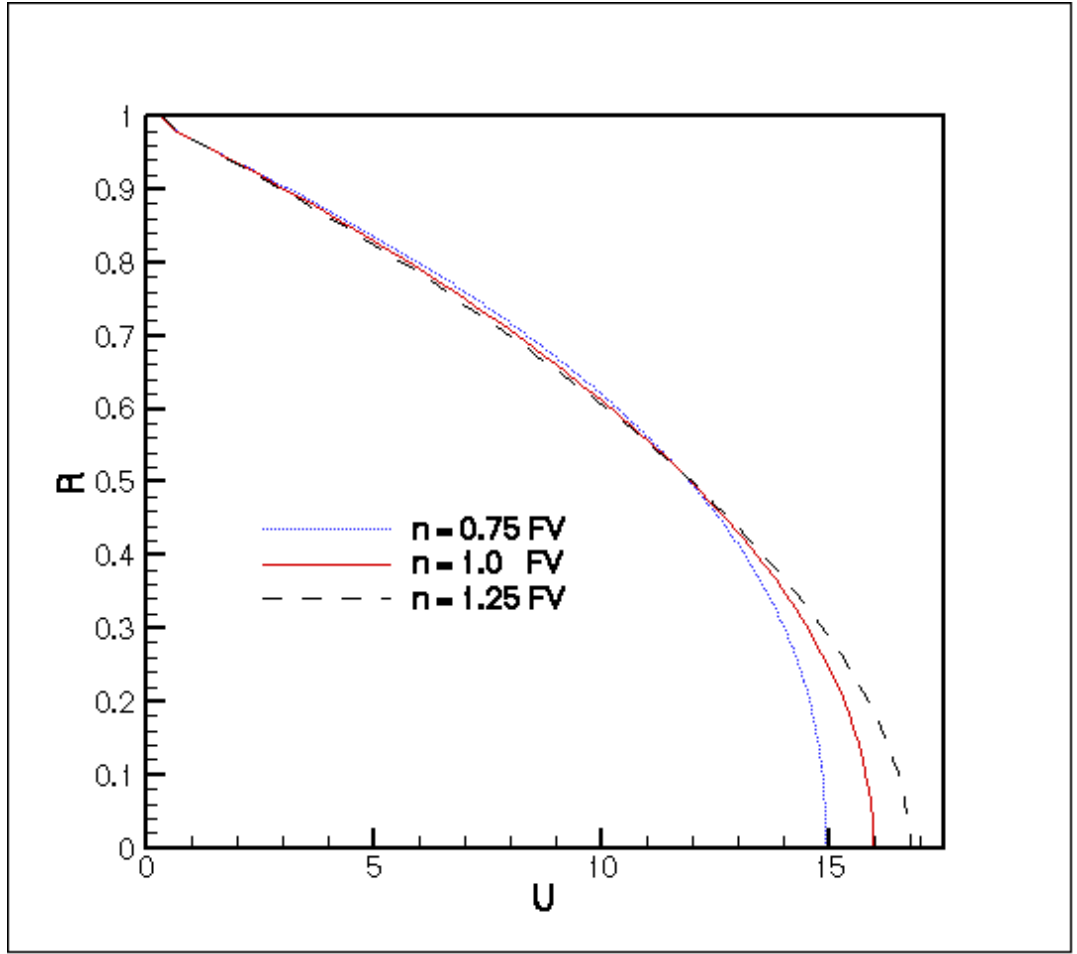

Fig. 16.- Perfil de la velocidad $u$ a la salida con MVF

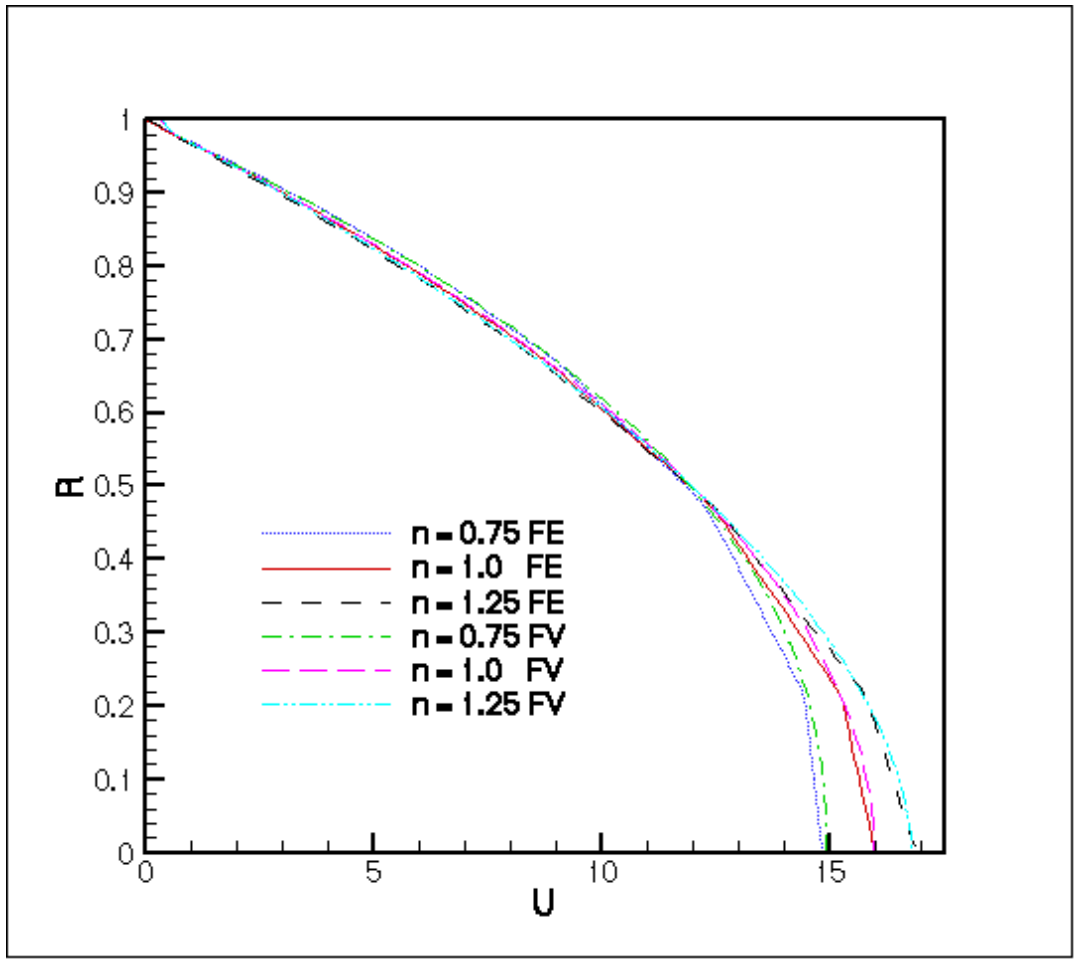

Fig. 17.- Comparación de los perfiles de la velocidad $u$ a la salida 


\section{CONCLUSIONES}

El movimiento de un fluido no newtoniano, tipo Ostwald de Waele, en una tubería circular con reducción brusca de diámetro, se describió empleando los métodos de elementos y de volúmenes finitos. Ambos métodos permiten calcular las distribuciones de velocidad y de presión para fluidos dilatantes, $\mathrm{n}<1$, fluidos newtonianos, $\mathrm{n}=1$, y fluidos pseudoplásticos, $\mathrm{n}>1$.

\section{AGRADECIMIENTOS}

Este trabajo se realizó gracias al financiamiento de FONDECYT, proyecto 1980032, y de DICYTUSACH.

\section{REFERENCIAS}

[1] J. N. Guerreiro, A. F. D. Loula; "Finite element analysis of transient creep problems", Comput. Methods Appl. Mech. Engnrg. Vol. 117, 1994.
[2] C. Taylor, P. Hood; "A numerical solution of the Navier - Stokes equations using the finite element technique. Computers and Fluids", 1:73-100, 1973.

[3] M. Crouzeix, P.A. Raviart; "Conforming and nonconforming finite elements methods for the stationary Stokes equation". Technical Report R3, RAIRO, 1973.

[4] J. Prusa; "Asymptotic and Numerical Solutions for Thermally Developing Flows of Newtonian and Non-Newtonian Fluids in Circular Tubes With Uniform Wall Temperature". Numerical Heat Transfer, Part A, 26:199-217, 1995.

[5] H. Huang, Z. Li, A. S. Usmani; "Finite Element Analysis of Non-Newtonian Flow". SpringerVerlag, 1999.

[6] C. Vial; "Estudio de Mecánica de Fluidos y Transferencia de Calor Transiente 2D en Ductos de Sección Transversal Variable para Fluidos no Newtonianos Mediante el Método de Volúmenes Finitos". Trabajo de Titulación de Ingeniería Civil Mecánica. Universidad de Santiago de Chile, 1996. 